\title{
Evaluación global subjetiva y escala de malnutrición- inflamación para valorar el estado nutricional de pacientes en diálisis peritoneal con hipoalbuminemia
}

\author{
Sagrario Jiménez Jiménez* - Francisca Muelas Ortega* - Pilar Segura Torres** - Francisco José Borrego Utiel** - \\ José Manuel Gil Cunquero** - Antonio Liébana Cañada**
}

*Enfermera. Unidad de Diálisis Peritoneal

**Facultativo Especialista de Nefrología

Servicio de Nefrología. Complejo Hospitalario de Jaén

\section{Resumen}

Los pacientes con insuficiencia renal crónica en diálisis peritoneal con frecuencia están mal nutridos o en riesgo de desnutrición, por sus especiales características y favorecido por múltiples factores, como otras patologías asociadas, diálisis inadecuada, estado inflamatorio, pérdida de nutrientes por el dializado, etc.

Se han descrito numerosos métodos para evaluar el estado nutricional, siendo las medidas antropométricas y determinaciones de laboratorio las más utilizadas, pero cuando las aplicamos a pacientes renales los resultados obtenidos son muy variables, además algunos de ellos plantean problemas a la hora de su aplicación, por las especiales características de los pacientes en diálisis.

Una forma de prevenir la malnutrición es identificar a aquellos pacientes en riesgo de desnutrición y evitar su deterioro progresivo. Nuestro objetivo, es evaluar el estado nutricional de los pacientes en diálisis peri-

Correspondencia:

Sagrario Jiménez Jiménez

C/ Travesía de San Joaquin 9. 4A. 23007. Jaén

E-mail: sagrariojj@hotmail.com toneal con hipoalbuminemia. Hemos seleccionado 21 pacientes con más de 3 meses en diálisis peritoneal y con tendencia a tener hipoalbuminemia $<3,6 \mathrm{~g} /$ dl. Hemos evaluado su estado nutricional mediante la escala de evaluación global subjetiva y escala de malnutrición-inflamación.

Hemos encontrado que la mayoría de los pacientes presentan bajo riesgo de malnutrición estimado tanto por la evaluación global subjetiva donde el 85,7\% tienen bajo riesgo de malnutrición o estado de nutrición normal y por la escala de malnutrición-inflamación igualmente el $85 \%$ tienen un estado de nutrición normal. Por tanto, debemos utilizar la combinación de varios métodos para evaluar adecuadamente el estado nutricional e identificar aquellos con riesgo de malnutrición.

\section{PALABRAS CLAVE:}

- DIÁLISIS PERITONEAL

- HIPOALBUMINEMIA

- MALNUTRICIÓN

- EVALUACIÓN GLOBAL SUBJETIVA

- ESCALA MIS 


\section{Subjective global assessment and malnutrition- inflammation scale to assess the nutritional status of patients on peritoneal dialysis with hypoalbuminaemia}

\section{Abstract}

Patients with chronic renal deficiency on peritoneal dialysis are often poorly nourished or at risk of malnutrition due to their special characteristics, and this is exacerbated by a range of factors, including other associated pathologies, unsuitable dialysis, inflammatory state, loss of nutrients due to the dialysate, etc.

Numerous methods for assessing nutritional status have been described, among which anthropometric measurements and laboratory determinations are the most widely-used. However, when these are applied to renal patients the results obtained are highly variable, and moreover problems arise with putting some of them into practice, due to the special features of dialysis patients.

One way of preventing malnutrition is to identify the patients at risk and avoid their gradual deterioration. The aim here is to assess the nutritional status of patients on peritoneal dialysis with hypoalbuminaemia. We selected 21 patients with over 3 months on peritoneal dialysis and with a tendency to suffer hypoalbuminaemia < $3.6 \mathrm{~g} / \mathrm{dl}$. We appraised their nutritional status using the subjective overall assessment scale and the malnutritioninflammation scale.

We found that most patients had a low estimated risk of malnutrition according to both the subjective global assessment where $85.7 \%$ had a low risk of malnutrition or a normal nutritional status and the malnutrition-inflammation scale, which likewise gave $85 \%$ with a normal nutritional status. We must therefore use a combination of different methods to properly assess nutritional status and identify those at risk of malnutrition.
KEY WORDS:
- PERITONEAL DIALYSIS
- HYPOALBUMINAEMIA
- MALNUTRITION
- SUBJECTIVE GLOBAL ASSESSMENT
- MIS SCALE

\section{Introducción}

En pacientes con insuficiencia renal crónica en diálisis es frecuente la malnutrición que ocasiona un aumento de la morbi-mortalidad ${ }^{1,2}$, mayor número y duración de los ingresos hospitalarios ${ }^{3}$ condicionando una baja calidad de vida ${ }^{1}$. En diálisis peritoneal (DP) la prevalencia de malnutrición parece ser elevada oscilando entre el 18$56 \%$ según diferentes estudios ${ }^{4,5}$.

Existen numerosos factores implicados en la malnutrición en DP como la escasa ingesta oral favorecida por múltiples factores como la edad, la pérdida de función renal residual, la diálisis inadecuada ${ }^{5}$; las enfermedades crónicas asociadas; el estado inflamatorio que favorece la anorexia y que genera catabolismo proteico; y la pérdida de nutrientes por el líquido dializado que aumentan cuando existen peritonitis ${ }^{5}$.

Una forma de prevenir la malnutrición es identificar a aquellos sujetos que se encuentren en riesgo nutricional, es decir, a aquéllos en los que existe probabilidad de que su situación nutricional se deteriore. Por eso, es importante realizar una valoración del estado nutricional de los pacientes y prestar especial atención en este aspecto a estos pacientes 6 . En ellos la intervención nutricional tendrá mayor probabilidad de éxito y buscará evitar el deterioro progresivo y permitir su recuperación, lo cual redundará en una reducción de los riesgos asociados a la malnutrición.

El amplio rango de malnutrición en DP viene dado por los diferentes métodos utilizados para valorar y clasificar el estado nutricional de los pacientes ${ }^{4}$. Entre ellos, son las medidas antropométricas y las determinaciones de laboratorio las más extendidas, pero cuando se aplican a pacientes renales, la prevalencia de malnutrición que dan es muy variada y su relación con el pronóstico también es muy variable, lo que quizás indique que no estén reflejando todas la misma información. Además, algunas de ellas plantean problemas en su aplicación en los pacientes en diálisis por sus especiales características, siendo muchos de ellos de difícil reproducción, por lo que la fiabilidad de los resultados no es completa ${ }^{6}$.

Aunque se han aplicado diversos métodos para evaluar el estado nutricional en pacientes en diálisis no existe claramente una técnica "gold standard". Unas son de aplicación clínica como la evaluación de la dieta, la antropometría y la evaluación global subjetiva y otras 
se basan en parámetros bioquímicos (albúmina, prealbúmina, colesterol, transferrina) o mezcla de ambos como la escala de malnutrición-inflamación (Escala MIS).

El peso corporal es una medida de evaluación nutricional básica y fácilmente disponible. Es una medida de salud global siendo reflejo entre otros de la suma del compartimento muscular y graso. En pacientes en diálisis presenta cierta variabilidad en el tiempo y es frecuente observar ascensos y descensos del peso de manera periódica o estacional, aunque sean pacientes estables. Descensos no intencionados del peso superiores al $5 \%$ en el último mes, al 7,5\% en 3 meses 0 al $10 \%$ en los últimos 6 meses deben considerarse como sugerentes de riesgo nutricional ${ }^{7}$.

La hipoalbuminemia es frecuente en pacientes en DP y depende del estado de hidratación. A su vez, hay algunos autores que sugieren que la expansión de volumen extracelular que presentan los pacientes en DP supone un estímulo para la inflamación, lo que motivaría mayor hipoalbuminemia, siendo ambas situaciones factores de riesgo independiente de mortalidad cardiovascular en $\mathrm{DP}^{8}$. Asimismo, los pacientes que llevan más tiempo en DP pierden función renal residual, presentan un transporte peritoneal elevado con mayor pérdida de proteínas, con fallo en la ultrafiltración (requiriendo utilización de hipertónicos o icodextrina y paso a técnicas automatizadas de DP), lo que se asocia a un estado hipervolemia e hipoalbuminemia?.

La evaluación global subjetiva (EGS) ${ }^{10}$ y escala de malnutrición-inflamación (MIS) ${ }^{11}$ están basadas en la combinación de de características subjetivas y objetivas de la historia clínica y de la exploración física y en la escala MIS además incluye parámetros analíticos. Ambas permiten clasificar a los pacientes según el riesgo de sufrir malnutrición creciente en los meses siguientes a la exploración.

El objetivo de este estudio fue, valorar el estado nutricional de pacientes en diálisis peritoneal con hipoalbuminemia.

\section{Material y Métodos}

Hemos seleccionado 21 pacientes en diálisis peritoneal que estuvieran estables en la técnica más de 3 meses y que en revisiones previas tuvieran tendencia a tener hipoalbuminemia (Albúmina $<3,6 \mathrm{~g} / \mathrm{dl}$ ). Para valorar el estado nutricional hemos utilizado la escala de evaluación global subjetiva y la escala MIS.

Para llevar a cabo dichas escalas se han realizado los siguientes parámetros:

- Cálculo del cambio de peso en los 3-6 meses previos

- Encuesta sobre ingesta dietética, sobre la cantidad y el tipo de alimentación (sólida, líquida...)

- Encuesta sobre síntomas gastrointestinales: náuseas, vómitos, etc.

- Datos sobre capacidad funcional relacionada con estado nutricional

- Comorbilidad asociada

- Realización de un examen físico para valorar la pérdida de los depósitos grasos y musculares de cabeza, tronco y extremidades.

- Cálculo del IMC

- Recogida de niveles de albúmina y transferrina

Para el cálculo de la Evaluación Global Subjetiva se clasificaran a los pacientes en tres categorías $A, B$ y $C$ teniendo en cuenta que la situación A sería compatible con la normalidad y la $C$ la situación extrema de desnutrición y la situación B sería la situación intermedia que mostraría algunos datos de desnutrición.

Para el cálculo de la Escala MIS se da una puntuación de 0 a 3 a cada uno de los apartados y se suma la puntuación total de tal manera que cuántos más puntos tenga un paciente mayor probabilidad de estar malnutrido-inflamado. El rango de puntuación va desde 0 a 30. Se considera normal tener una puntuación inferior a 8 puntos.

\section{Resultados}

Tenemos un total 21 pacientes cuya edad media es $57,4 \pm 18,4$ años. Existe un predominio de mujeres que suponen el $52,4 \%$. El 23,8\% son diabéticos. En DPCA hay 16 pacientes y en DPA hay 5 pacientes. EI tiempo medio en DPCA es $24,8 \pm 24,1$ meses y en DPA es $13,8 \pm 24,4$ meses. Los niveles medios de albúmina 
son $3,5 \pm 0,3 \mathrm{~g} / \mathrm{dl}$. A la exploración física presentan edemas el $42,9 \%$.

Si consideramos la Evaluación Global Subjetiva (Tabla I) encontramos que la puntuación total de cada categoría fue $\mathrm{A} 85,7 \%$, B $14,3 \%$ y C $0 \%$ de los pacientes. Con respecto a cada item por separado tenemos:

- En la variación de peso el 90,5\% están en la categoría A y el 9,5\% en la categoría $B$, nadie en la categoría C.

- Respecto a la ingesta dietética el $100 \%$ se encuentran en la categoría A.

- En los síntomas gastrointestinales el 90,5\% están en la categoría $A$ y el $9,5 \%$ en la categoría $B$, nadie en la categoría C. El tener síntomas gastrointestinales parece que se asocia a mayor probabilidad de malnutrición moderada, puesto que cuando presentan síntomas gastrointestinales el $100 \%$ están en categoría $\mathrm{B}$ y cuando no existen síntomas gastrointestinales solo el 5,3\% están en categoría $B ; p<0,001$.

- Referente a la capacidad funcional el $81 \%$ están en la categoría $A$, el 9,5\% en la categoría $B$ y el 9,5\% en la categoría $C$.

- La comorbilidad asociada, el 76,2\% están en la categoría $A$ y el $23,8 \%$ en la categoría $B$, nadie en la categoría $\mathrm{C}$.

- Con respecto a los signos de pérdida de los depósitos de grasa subcutánea, el 85,7\% están en la categoría A y el $14,3 \%$ en la categoría $B$, nadie en la categoría $C$.

- En los signos de pérdida de la masa muscular el $76,2 \%$ están en la categoría A, el 19\% están en la categoría $B$ y el $4,8 \%$ están en la categoría $C$.

Respecto a la puntuación total de la escala MIS (Tabla II), encontramos que el $85 \%$ tienen una puntuación inferior o igual a 8 puntos, el $5 \%$ tiene 9 puntos, otro $5 \%$ tiene 13 puntos y otro 5\% tiene 18 puntos. Si analizamos por separado cada item encontramos que:

- El 38,1\% no perdieron peso o la pérdida de peso fue inferior a $0,5 \mathrm{~kg}$, el $23,8 \%$ la pérdida de peso fue entre $0,5-1 \mathrm{~kg}$, en el $33,3 \%$ la pérdida de peso fue mayor de $1 \mathrm{~kg}$ y menor del $5 \%$ y el $4,8 \%$ la pérdida de peso fue mayor del $5 \%$.

- En la ingesta dietética el $71,4 \%$ tenían 0 puntos y el $28,6 \%$ tenían 1 punto.
- Respecto a los síntomas gastrointestinales el 71,4\% tenían 0 puntos, el 19,1\% tenían 1 punto y el 9,5\% tenían 2 puntos.

- Con la capacidad funcional, el $66,7 \% 0$ puntos, $14,3 \%$ tenían 1 punto, el 9,5\% tenían 2 puntos y el 9,5\% tenían 3 puntos.

- En la comorbilidad el $42,9 \%$ tenían 0 puntos, el $33,3 \%$ tenían 1 punto y el 23,8\% tenían 2 puntos.

- Con respecto a los signos de pérdida de los depósitos grasos el $85,7 \%$ tenían 0 puntos y el 14,3\% tenían 2 puntos.

- En los signos de pérdida de los depósitos musculares el $76,2 \%$ tenían 0 puntos, el $19 \%$ tenían 2 puntos y el 4,8\% tenían 3 puntos.

- El IMC en el $100 \%$ es de 0 puntos.

- Los niveles de albúmina, el $0 \%$ tenían 0 puntos, el $65 \%$ tenían 1 punto, el 30\% tenían 2 puntos y el $5 \%$ tenían 3 puntos.

- Los niveles de transferrina, el 55\% tenían 0 puntos, el $40 \%$ tenían 1 punto y el 5\% tenían 3 puntos.

\section{Discusión}

Ante la variable prevalencia de malnutrición en pacientes en DP y su asociación con mayor morbi-mortalidad, es necesario poder identificar a los pacientes malnutridos para establecer una adecuada intervención nutricional.

La hipoalbuminemia, que es un parámetro utilizado tradicionalmente en la evaluación nutricional, ha pasado a considerarse hoy como un parámetro más reflejo de la inflamación que del verdadero estado nutricional de un sujeto. Sus niveles en sangre se ven rápida y fácilmente influenciados por los cambios inflamatorios y del estado de hidratación lo que obliga a buscar otros métodos de valoración nutricional.

Tras seleccionar pacientes en diálisis peritoneal con hipoalbuminemia y valorar el estado nutricional mediante la utilización de la evaluación global subjetiva y de la escala MIS, encontramos que la mayoría de los pacientes en diálisis peritoneal que tienen niveles de albúmina bajos presentan bajo riesgo de malnutrición estimado tanto por la evaluación global subjetiva donde el $85,7 \%$ tienen bajo riesgo de malnutrición o estado 


\begin{tabular}{|l|c|c|c|}
\hline & Categoría A & Categoría B & Categoría C \\
\hline Pérdida de peso & $90,5 \%$ & $9,5 \%$ & $0 \%$ \\
\hline Ingesta dietética & $100 \%$ & $0 \%$ & $0 \%$ \\
\hline Síntomas gastrointestinales & $90,5 \%$ & $9,5 \%$ & $0 \%$ \\
\hline Capacidad funcional & $81 \%$ & $9,5 \%$ & $9,5 \%$ \\
\hline Comorbilidad asociada & $76,2 \%$ & $23,8 \%$ & $0 \%$ \\
\hline Depósitos grasos & $85,7 \%$ & $14,3 \%$ & $0 \%$ \\
\hline Depósitos musculares & $76,2 \%$ & $19 \%$ & $4,8 \%$ \\
\hline Total & $85,7 \%$ & $14,3 \%$ & $0 \%$ \\
\hline
\end{tabular}

Tabla 1. Evaluación global subjetiva

de nutrición normal y al estimarlo por la escala MIS igualmente el $85 \%$ tienen un estado de nutrición normal. Este mismo resultado fue obtenido por el grupo de Manzano ${ }^{12}$ llevado a cabo en un centro periférico en 2004 en pacientes en hemodiálisis, que pretendían describir el estado nutricional de los pacientes y que encontraron que el $85,1 \%$ presentaban un estado nutricional normal. En estos pacientes los niveles bajos de albúmina serían más el reflejo de un estado de inflamación y/o hiperhidratación que de malnutrición propiamente dicho, como pone también de manifiesto el estudio realizado por Manzano ${ }^{12}$ en el que para valorar el estado nutricional de los pacientes utiliza medidas antropométricas, marcadores bioquímicos y parámetros clínicos y concluyeron que la hipoalbuminemia tiene un origen multifactorial y que puede estar relacionada también con factores no nutricionales como situaciones comórbidas e inflamación ${ }^{12}$.

Entre los factores que determinan la concentración de albúmina plasmática en pacientes en diálisis peritoneal se encuentra el balance entre la síntesis hepática y su catabolismo, las pérdidas a través del efluente peritoneal y el volumen de distribución ${ }^{6}$. El estado de hipervolemia circulante puede ocasionar hipoalbuminemia simplemente por efecto dilucional de las proteínas. En este sentido, el estado de hiperhidratación es uno de los factores no nutricionales implicados en los bajos

\begin{tabular}{|c|c|c|c|c|}
\hline & 0 puntos & 1 punto & 2 puntos & 3 puntos \\
\hline Pérdida de peso & $38,1 \%$ & $23,8 \%$ & $33,3 \%$ & $4,8 \%$ \\
\hline Ingesta dietética & $71,4 \%$ & $28,6 \%$ & $0 \%$ & $0 \%$ \\
\hline Síntomas gastrointestinales & $71,4 \%$ & $19,1 \%$ & $9,5 \%$ & $0 \%$ \\
\hline Capacidad funcional & $66,7 \%$ & $14,3 \%$ & $9,5 \%$ & $9,5 \%$ \\
\hline Comorbilidad asociada & $42,9 \%$ & $33,3 \%$ & $23,8 \%$ & $0 \%$ \\
\hline Depósitos grasos & $85,7 \%$ & $14,3 \%$ & $0 \%$ & $0 \%$ \\
\hline Depósitos musculares & $76,2 \%$ & $19 \%$ & $4,8 \%$ & $0 \%$ \\
\hline IMC $\left(\mathrm{kg} / \mathrm{m}^{2}\right)$ & $100 \%$ & $0 \%$ & $0 \%$ & $0 \%$ \\
\hline Albúmina (g/dl) & $0 \%$ & $65 \%$ & $30 \%$ & $5 \%$ \\
\hline \multirow[t]{2}{*}{ Transferrina (mg/dl) } & $55 \%$ & $40 \%$ & $0 \%$ & $5 \%$ \\
\hline & $\leq 8$ puntos & 9 puntos & 13 puntos & 18 puntos \\
\hline Total & $85 \%$ & $5 \%$ & $0 \%$ & $5 \%$ \\
\hline
\end{tabular}

Tabla 2. Escala de Malnutrición-inflamación (MIS) 
niveles de albúmina y que podemos poner de manifiesto simplemente con la exploración clínica, demostrando la presencia de edemas, que en nuestro caso estaba presente en el $42,9 \%$ de los pacientes.

Ningún paciente presenta un riesgo elevado de desnutrición por ninguna escala. No hay ningún paciente en categoría $C$ de evaluación global subjetiva y con respecto a la escala MIS son pocos los pacientes que se encuentran por encima del punto de corte normal y están en torno a dicho punto, existiendo poca dispersión de los datos.

Cuando hemos considerado cada item por separado el resultado sigue siendo en el mismo sentido, estando la mayoría de los pacientes con un riesgo bajo de malnutrición por ambas escalas. Con respecto a los síntomas gastrointestinales parece que es un factor al que habría que prestar especial atención en la práctica clínica diaria puesto que los pacientes que tienen molestias gastrointestinales presentan mayor probabilidad de presentar un moderado riesgo de malnutrición. Así se sabe, que los pacientes en diálisis peritoneal comen poco y podemos observar como disminuye el apetito y la ingesta de proteínas a partir de los 12 primeros meses en la técnica ${ }^{13}$. La causa primaria de esta pobre ingesta suele ser la anorexia que en los pacientes con insuficiencia renal crónica puede ser debida a distintos factores siendo el más importante la toxicidad urémica por diálisis insuficiente cuya principal manifestación es digestiva en forma de náuseas y vómitos ${ }^{6}$.

En general, parece que predomina la malnutrición proteica derivada de la baja ingesta y de las perdidas proteicas por el dializado. Aunque la ingesta calórica también está reducida, pero se ve mantenida gracias a la absorción de glucosa del líquido peritoneal, encontrando que los parámetros que miden el compartimento de grasa pueden ser normales, sin embargo, el compartimento proteico medido tanto en determinaciones bioquímicas como antropométricas se encuentran por debajo de lo normal6. En nuestro estudio también podemos observar hallazgos en este sentido, puesto que hemos encontrado que no hay ningún paciente que muestre signos extremos de pérdida de depósitos grasos mientras que el 4,8\% muestran signos extremos de pérdida del compartimento muscular.

Podemos concluir que el riesgo de malnutrición de pacientes con hipoalbuminemia en diálisis peritoneal es bajo, medido con la escala de evaluación global subjetiva y la escala MIS, por lo que debemos utilizar la combinación de varios métodos para evaluar adecuadamente el estado nutricional e identificar aquellos con riesgo de malnutrición.

Recibido: 14 Marzo 2012

Revisado: 30 Marzo 2012

Modificado: 15 Abril 2012

Aceptado: 20 Abril 2012

\section{Bibliografía}

1. Sánchez S, Fdez. Reyes MJ, Celadilla 0, Martínez MV, Muñoz I, Rodrigo N et al. Nutrición en pacientes de dialysis peritoneal. Un reto para enfermería. Estudio transversal. Rev Soc Esp Enferm Nefrol 1996; 1996(4): Biseden IV trimestre 1996.

2. Chung $S H$, Lindholm $B$, Lee HB. Is malnutrition an independent predictor of mortality in peritoneal dialysis patients? Nephrol Dial Transplant. 2003 0ct;18(10):2134-40.

3. Young G.A., Kopple J.D., Lindholm B., Vonesh E. F., De Vecchi A., et al. Nutritional assessment of continuous ambulatory peritoneal dialysis patients: an international study. Am J Kidney Dis XVII (4): 462-471, 1991.

4. Dombros NV, Digenis GE, Oreopoulos DG. Is Malnutrition a problem for the patient on peritoneal dialysis? Perit Dial Int 1995; 15;(Suplement 5): S10-S19. 1995.

5. Piraino B. Recommendations for dietary protein intake in CAPD patients. Adv Perit Dial. 1996; 12:275-279.

6. Guerrero Risco A. Nutrición y diálisis adecuada en diálisis peritoneal. Rev Soc Esp Enferm Nefrol 1999; 2(1).

7. Pifer T.B., McCullough K.P., Port F.K., Goodkin D.A., Maroni B.J., Held Ph.J. y Young E.W. Mor- 
tality risk in hemodialysis patients and changes in nutritional indicators: DOPPS. Kidney Int 62: 2238-2245, 2002.

8. Avila-Díaz $M$, Ventura MD, Valle $D$, Vicente-Martínez M, García-González Z, Cisneros A, et al. Inflammation and extracellular volume expansion are related to sodium and water removal in patients on peritoneal dialysis. Perit Dial Int 2006; 26(5):574580.

9. Tzamaloukas AH. Risk of extracellular volume expansion in long-term peritoneal dialysis. Adv Perit Dial. 2005;21:106-111.

10. Cooper B.A., Bartlett L.H., Aslani A., Allen B.J. Ibels LI.S., Pollock C.A. Validity of subjective global assessment as a nutritional marker in end-sta- ge renal disease. Am J Kidney Dis 40(1): 126-132, 2002.

11. Kalantar-Zadeh K., Kopple J.D., Humphreys M.H. y Block G. Comparing outcome predictibility of markers of malnutrition-inflammation complex syndrome in haemodialysis patients. Nephrol Dial Transplant 19: 1507-1519, 2004.

12. Manzano JM, Nieto MD, Sánchez MC. Valoración nutricional de enfermería de los pacientes tratados con hemodiálisis en un centro periférico. Rev Soc Esp Enferm Nefrol 2004; 7 (1): 10-18

13. Hylander B, Barkeling B, Rossner S. Eating behavior in continuous peritoneal dialysis and hemodialysis patients. Am Soc Kidney Dis; 20: 592-597. 1992 